\title{
American Joint Committee on Cancer
}

National Cancer Institute

\section{Source}

National Cancer Institute. American Joint Committee on Cancer. NCI Thesaurus. Code C39315.

A group formed for the purpose of developing a system of clinical staging for cancer that is acceptable to the American medical profession and is compatible with other accepted classifications. 